\title{
Generalized net models of queueing disciplines in finite buffer queueing systems with intuitionistic fuzzy evaluations of the tasks
}

\author{
Zhivko Tomov ${ }^{1}$, Maciej Krawczak ${ }^{2}$, Velin Andonov ${ }^{3}$, \\ Krassimir Atanassov ${ }^{1,4}$ and Stanislav Simeonov ${ }^{1}$ \\ 1 "Prof. Dr Asen Zlatarov" University \\ 1 Yakimov Blvd., Burgas 8000, Bulgaria \\ e-mail: zhivko57@yandex.ru, st_sim@yahoo.com \\ ${ }^{2}$ Systems Research Institute, Polish Academy of Sciences \\ Newelska 6, 01-447 Warsaw, Poland \\ e-mail: krawczak@ibspan.waw.pl \\ ${ }^{3}$ Institute of Mathematics and Informatics, Bulgarian Academy of Sciences \\ Acad. G. Bonchev Str., Block 8, Sofia 1113, Bulgaria \\ e-mail: velin_andonov@math.bas.bg
}

${ }^{4}$ Institute of Biophysics and Biomedical Engineering, Bulgarian Academy of Sciences

Acad. G. Bonchev Str., Block 105, Sofia 1113, Bulgaria

email: krat@bas.bg

Received: 1 February 2019

Revised: 28 March 2019

Accepted: 9 April 2019

\begin{abstract}
Generalized net models of different queueing disciplines in queueing systems are proposed in [11]. In the present paper, we propose modifications of these models including Intuitionistic Fuzzy Pairs (IFP) and Interval-Valued Intuitionistic Fuzzy Pairs (IVIFP) which determine the way in which the requests are serviced. In each of the models, the buffer has finite capacity and is represented by two Generalized net transitions. The buffer cells are represented by places of the net. The two simple queueing disciplines considered are FIFO and LIFO. A more general model with IFP (or IVIFP) in which the requests can change their parameters and position within the buffer is also proposed.
\end{abstract}

Keywords: Generalized net, Queueing system, Queueing discipline, Intuitionistic fuzzy pair. 2010 Mathematics Subject Classification: 68Q85, 03E72. 


\section{Introduction}

The present paper is a continuation of our research on the conceptual modelling of queuing systems presented in [11]. As it is mentioned there, the idea of modelling of the processes in buffers (entering and leaving of requests/pieces of information) by Petri nets lead to the introduction of the Buffer-Transition Nets of Hans Fuss [6]. They represent exactly the buffer functioning, following the procedures of queueing theory (see, e.g., [7-10]).

In [11], Generalized Net (GN) models of these processes are described and some new ones are introduced. For the definition of GN and the concepts of the GNs theory the reader can refer to $[3,4]$.

For brevity, here we use only reduced GNs without priorities of the transitions, places and tokens, without temporal components and capacities of the places corresponding to the buffer cells equal to 1, while the capacities of the rest of the places are not specified, i.e., they can be $\infty$. The arcs capacities and the transition types are not defined.

Let us have the source of data $G$ (generator of pieces of information/requests), buffer $B$ with $n$ cells $b_{1}, \ldots, b_{n}$ and Server denoted by $U$ (see Figure 1 ).

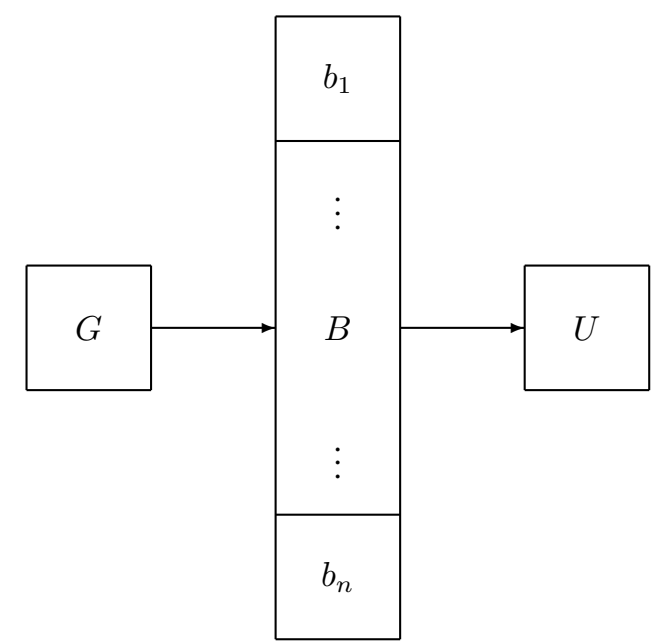

Figure 1. Conceptual representation of a buffer.

As it is mentioned in [11], the GN model of a queueing system in service networks with FIFO queueing discipline is represented by a GN containing only two transitions (see Figure 2), where as above, $n$ is the number of buffer cells (capacity of the buffer), that are represented here by GN-places.

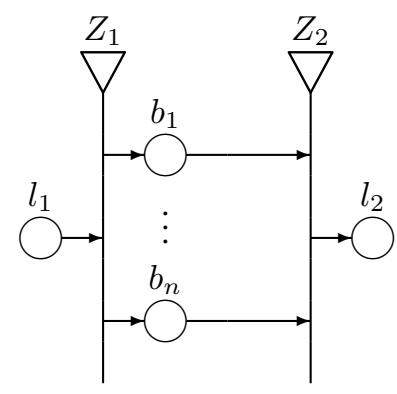

Figure 2. GN representation of simple queueing disciplines. 
The transitions of the GN shown in Figure 2 have the following formal description:

$$
Z_{1}=\left\langle\left\{l_{1}\right\},\left\{b_{1}, \ldots, b_{n}\right\}, r_{1}\right\rangle
$$

where

$$
r_{1}=\begin{array}{c|ccccc} 
& b_{1} & \ldots & b_{j} & \ldots & b_{n} \\
\hline l_{1} & r_{1,1} & \ldots & r_{1, j} & \ldots & r_{1, n}
\end{array} ;
$$

and $r_{1, j}=$ " $b_{j}$ is the first empty place from top to bottom among places $b_{1}, b_{2}, \ldots, b_{n}$ ", where $1 \leq j \leq n$.

$$
Z_{2}=\left\langle\left\{b_{1}, \ldots, b_{n}\right\},\left\{l_{2}\right\}, r_{2}\right\rangle
$$

where

$$
r_{2}=\begin{array}{c|c} 
& l_{2} \\
\hline b_{1} & s_{1,2} \\
\vdots & \vdots \\
b_{j} & s_{j, 2} \\
\vdots & \vdots \\
b_{n} & s_{n, 2}
\end{array}
$$

and $s_{j, 2}=$ "the token in place $b_{j}$ has stayed for more time in the place compared to the tokens in all places $b_{1}, b_{2}, \ldots, b_{n} "$, for $j=1,2, \ldots, n$.

In this way, we describe the queueing discipline "first enters - first comes out" (FIFO).

The second GN model, describing the other simple queueing discipline, namely, "last enters - first comes out" (LIFO) has the same graphical representation as in Figure 2 and the difference is only in the form of the predicates, which are now, respectively, $r_{1, j}=$ " $b_{j}$ is the first empty place from top to bottom among places $b_{1}, b_{2}, \ldots, b_{n}$ ", for $j=1,2, \ldots, n, s_{j, 2}=$ "the token in place $b_{j}$ has stayed in the place for less time compared to the tokens in places $b_{1}, b_{2}, \ldots, b_{n}$ ", for $j=1,2, \ldots, n$.

In [11], more than ten GN models of different queueing disciplines in a queueing system were proposed. In none of them, however, elements of fuzziness are included. In the present paper, we show possibilities to add such elements in the GN models.

\section{Main results}

Let each piece of information/requests (represented by an $\alpha$-token in the GN model) has an initial characteristic

$$
\text { "respective information/requests; } \mu_{\alpha}, \nu_{\alpha} ",
$$

where $\mu_{\alpha}, \nu_{\alpha} \in[0,1]$ and $\mu_{\alpha}+\nu_{\alpha} \leq 1$

The ordered pair $\left\langle\mu_{\alpha}, \nu_{\alpha}\right\rangle$ is called Intuitionistic Fuzzy Pair (IFP) (see [5]).

The ordered pair $\langle M, N\rangle$ where $M, N \subseteq[0,1]$ are closed sets is called Interval-Valued Intuitionistic Fuzzy Pair (IVIFP) if $M=[\inf M, \sup M], N=[\inf N, \sup N]$ and

$$
\sup M+\sup N \leq 1 \text {. }
$$


The IFP (IVIFP) can be interpreted as degrees of validity and of non-validity of the information or as degree of correctness and non-correctness of the request.

With this in mind, the above two GN models can be modified in the following way.

In the first GN model we keep the form of predicate $r_{1, j}$ and change predicate $s_{j, 2}$, for $j=1,2, \ldots, n$ to one of the following three forms:

- $s_{j, 2}=$ "the token in place $b_{j}$ has the highest $\mu$-value in comparison with the other tokens in the places $b_{1}, b_{2}, \ldots, b_{n}$ "; or

- $s_{j, 2}=$ "the token in place $b_{j}$ has the lowest $\nu$-value in comparison with the other tokens in the places $b_{1}, b_{2}, \ldots, b_{n}$ "; or

- $s_{j, 2}=$ "the token in place $b_{j}$ has highest $\langle\mu, \nu\rangle$-value in comparison with the other tokens in the places $b_{1}, b_{2}, \ldots, b_{n}$ ",

where for two IFPs $\left\langle\mu_{1}, \nu_{1}\right\rangle$ and $\left\langle\mu_{2}, \nu_{2}\right\rangle$ we say that $\left\langle\mu_{1}, \nu_{1}\right\rangle$ is higher than $\left\langle\mu_{2}, \nu_{2}\right\rangle$ if and only if

$$
\mu_{1} \geq \mu_{2} \quad \text { and } \quad \nu_{1} \leq \nu_{2}
$$

Obviously, the first two forms of these predicates are weaker forms of the third one.

In the second GN model (shown in Figure 2) we associate with each place $b_{j}$ among places $b_{1}, b_{2}, \ldots, b_{n}$ the IVIFP $\left\langle M_{j}, N_{j}\right\rangle$. Now, predicate $r_{1, j}$ for $j=1,2, \ldots, n$ has one of the following three forms for the current $\alpha$-token in place $l_{1}$ :

- $r_{1, j}=$ " $\mu_{\alpha} \in M_{j}$ "; or

- $r_{1, j}=$ " $\nu_{\alpha} \in N_{j}$ "; or

- $r_{1, j}=" \mu_{\alpha} \in M_{j} " \& " \nu_{\alpha} \in N_{j} "$

Obviously, again, the first two predicates are weaker forms of the third one.

This modification is an example where the Generalized Nets with Characteristics of the Places (GNCP, see [1]) can be used. If the IVIFPs, associated with the respective places, do not change during the functioning of the GN, then we can say that this model is static. It can obtain a dynamic form, if the IVIFPs change with time. This can be realized with the help of the characteristic functions of the places, described in [1].

The situation with the output place $l_{2}$ is similar. If there is an IVIFP $\langle M, N\rangle$ associated with it, then the predicate $s_{j, 2}$ for $j=1,2, \ldots, n$ has one of the following three forms for the current $\alpha$-token in place $l_{j}$ :

- $s_{j, 2}=$ " $\mu_{\alpha} \in M$ "; or

- $s_{j, 2}=$ " $\nu_{\alpha} \in N$ "; or

- $s_{j, 2}=" \mu_{\alpha} \in M " \& " \nu_{\alpha} \in N "$. 
In this case, probably, some $\alpha$-tokens will stay permanently in their places, because for them the corresponding predicate $s_{j, 2}$ will be permanently false. So, the places' characteristic must be changed during the functioning of the net through the characteristic function of the place.

The two types of GNs models can be united in one GN model with the above new forms of the predicates $r_{1, j}$ and $s_{j, 2}$ for $j=1,2, \ldots, n$.

In the above GN models we are not interested in the characteristics of the tokens entering $l$ and $b$-places. They are assigned to the tokens by the generator of data and the tokens keep them without changes during their transfer in the GN.

Similarly to [11], in order to determine the duration of staying of the tokens in places $b_{1}, b_{2}, \ldots$, $b_{n}$, we can consider that upon entering the places corresponding to the buffer cells, the tokens obtain as characteristic the time-moment of entering.

Now, all GN models in [11] can be modified in the way discussed above to include elements of fuzziness. By doing so we obtain GN models with additional criteria for tokens' transfer. In the simpler form of these GN models, the criteria are fixed, while in the extended model the additional criteria for transfer of tokens can be changed during the functioning of the net.

While, as we mentioned above, the IVIFPs associated with the places can be changed with the characteristic functions of the places, the $\langle\mu, \nu\rangle$-values of the tokens are constant.

Here, we modify the seventh GN model from [11] including elements of fuzziness.

In the new GN model, the tokens can change their places within the buffer (the places $b_{1}, b_{2}, \ldots$, $b_{n}$ ), according to some criterion $D$. This GN is shown in Figure 3. In some moments, token $\delta$ will enter place $d_{1}$ with initial characteristic $x_{o}^{\delta}=$ "criterion D for change of the tokens' places in the buffer".

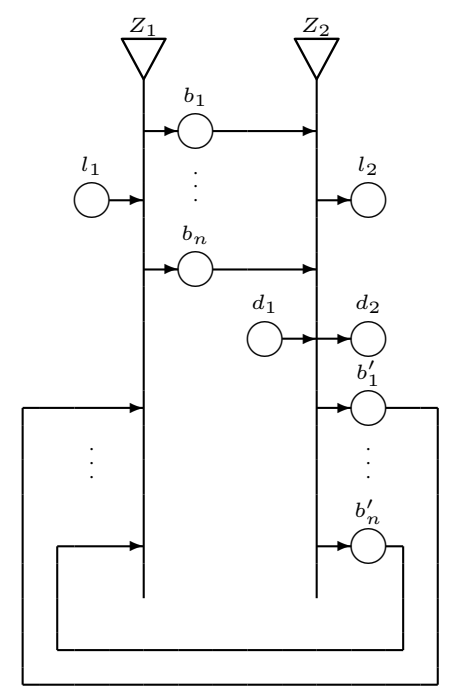

Figure 3. GN representation of queueing discipline with requests changing their positions within the buffer.

Now, the GN-transitions have the forms:

$$
Z_{1}=\left\langle\left\{l_{1}, b_{1}^{\prime}, \ldots, b_{n}^{\prime}\right\},\left\{b_{1}, \ldots, b_{n}\right\}, r_{1}\right\rangle
$$

where 


$$
\begin{array}{c|ccccc} 
& b_{1} & \cdots & b_{j} & \cdots & b_{n} \\
\hline l_{1} & r_{1,1} & \cdots & r_{1, j} & \cdots & r_{1, n} \\
b_{1}^{\prime} & t_{1,1} & \cdots & t_{1, j} & \cdots & t_{1, n} \\
\vdots & \vdots & \ddots & \vdots & \ddots & \vdots \\
b_{i}^{\prime} & t_{i, 1} & \cdots & t_{i, j} & \cdots & t_{i, n} \\
\vdots & \vdots & \ddots & \vdots & \ddots & \vdots \\
b_{n}^{\prime} & t_{n, 1} & \cdots & t_{n, j} & \cdots & t_{n, n}
\end{array}
$$

and $r_{1, j}$ for $j=1,2, \ldots, n$ has one of the above discussed forms and $t_{i, j}=$ "following criterion $D$, specified by the characteristic $x_{0}^{\delta}$, the current token has to be transferred from place $b_{i}^{\prime}$ to place $b_{j}$ ", where $1 \leq i \leq n$ and $1 \leq j \leq n$.

Upon entering an output place the $\alpha$-tokens obtain the characteristic:

$$
\text { " }\left\langle\mu_{\alpha}^{\text {new }}, \nu_{\alpha}^{\text {new }}\right\rangle "
$$

i.e., new truth-values are assigned to them. In this way, at some future time-moment they can satisfy the predicate that determines their transfer to place $l_{2}$.

$$
Z_{2}=\left\langle\left\{b_{1}, \ldots, b_{n}, d_{1}\right\},\left\{l_{2}, d_{2}, b_{1}^{\prime}, \ldots, b_{n}^{\prime}\right\}, r_{2}\right\rangle,
$$

where

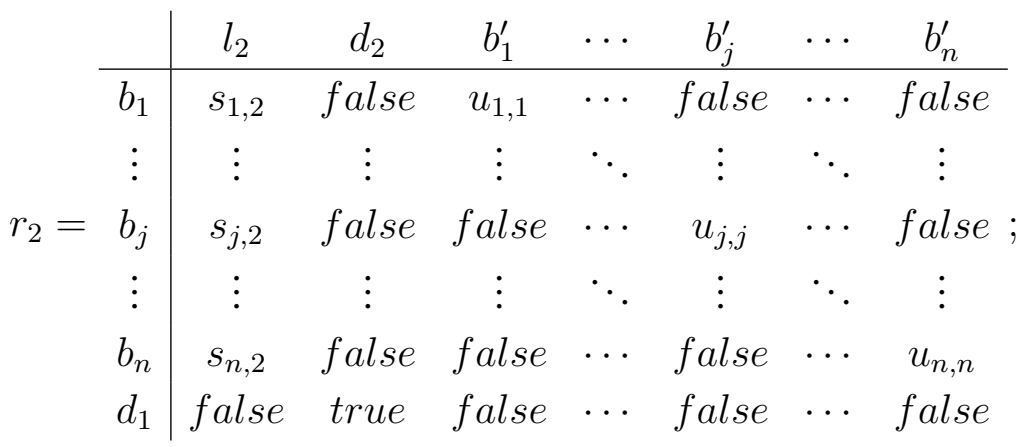

and $s_{j, 2}$ has one of the above discussed forms and $u_{j, j}=$ "following criterion $D$ specified by the characteristic $x_{0}^{\delta}$, the current token has to be transferred from place $b_{j}$ to another place".

All of the other GN models of finite buffer queueing systems from [11] can be modified in a similar way to include IFP (IVIFP) which determine the service of the requests in the buffer. In the case of GNs with tokens having duration of life, the modified model of the queueing system, including intuitionistic fuzzy parameters, can be used in the conceptual models of overall telecommunication networks. It is important to compare this model with the conceptual models of queueing systems in service networks discussed in [10].

\section{Conclusion}

As a continuation of the present paper we shall propose GN models of other queueing systems such as queueing systems with feedback, with catastrophes, failures and repairs, including IFP 
and IVIFP determining the transfer of the tokens. The models proposed in the present paper and in [11] will be adapted to the case of Markov's chain.

In [12], different GNs are described as tools for the modelling of Data Mining (DM) processes. Having in mind that the queueing systems can be included in the DM tools, the present paper is a continuation of the research discussed there.

The construction of a GN model of a queueing system with tokens duration of life is an important step towards the development of a conceptual model of overall telecommunication network with queueing system in the switching stage. It is interesting to compare such model to the one proposed in [2].

\section{Acknowledgements}

The authors from "Prof. Asen Zlatarov" University are thankful for the support provided by the European Regional Development Fund through the Operational Programme "Science and Education for Smart Growth" under contract UNITe No. BG05M2OP001-1.001-0004 (20182023). The author from the Institute of Mathematics and Informatics, Bulgarian Academy of Sciences, is thankful for the support provided by the National Scientific Fund of Bulgaria under Grant DM 12/2 "New Models of Overall Telecommunication Networks with Quality of Service Guarantees".

\section{References}

[1] Andonov, V., Atanassov, K. (2013). Generalized nets with characteristics of the places. Compt. rend. Acad. bulg. Sci., 66 (12), 1673-1680.

[2] Andonov, V., Poryazov, S., Otsetova, A., \& Saranova, E. (2019). A Queue in Overall Telecommunication System with Quality of Service Guarantees. Proc. of 4th EAI International Conference on Future Access Enablers of Ubiquitous and Intelligent Infrastructures (FABULOUS'2019), Sofia, 28-29 March, 2019 (in press).

[3] Atanassov, K. (1991). Generalized Nets, Singapore, World Scientific.

[4] Atanassov, K. (2007). On Generalized Nets Theory, "Prof. M. Drinov" Academic Publishing House, Sofia.

[5] Atanassov, K., Szmidt, E., \& Kacprzyk, J. (2013). On intuitionistic fuzzy pairs. Notes on Intuitionistic Fuzzy Sets, 19 (3), 1-12.

[6] Fuss, H. (1975). P-t-netze zur numerischen simulation von asynchronen fluessen. In: $J$. Hartmanis G. Goos, editor, GI - 4. Jahrestagung, Berlin 0.-12. Oktober 1974, number 26 in Lecture Notes in Computer Science, 326-335, GI, Gesellschaft fuer Informatik, SpringerVerlag. 
[7] Gross, D., Shortle, J., Thompson, J., \& Harris, C. (2008). Fundamentals of Queueing Theory, 4th edition. John Wiley \& Sons, New York.

[8] Kleinrock, L. (1975). Queueing Systems, Vol. I: Theory. Wiley, New York.

[9] Kleinrock, L. (1976). Queueing Systems, Vol. II: Computer Applications. Wiley, New York.

[10] Poryazov, S., Andonov, V. \& Saranova, E. (2018). Comparison of four conceptual models of a queueing system in service networks. Proc. of 26th National Conference with International Participation TELECOM'2018), Sofia, 25-26 October 2018, 71-77.

[11] Tomov, Zh., Krawczak, M., Andonov, V., Dimitrov, E., \& Atanassov, K. (2018). Generalized net models of queueing disciplines in finite buffer queueing systems. Proceedings of 16th International Workshop on Generalized Nets, 10 Feb. 2018, Sofia, 1-9.

[12] Zoteva, D., \& Krawczak, M. (2017). Generalized nets as a tool for the modelling of data mining processes. A survey. Issues in Intuitionistic Fuzzy Sets and Generalized Nets, 13, $1-60$. 